\title{
Kinases SPAK and OSR1 Are Upregulated by Estradiol and Activate NKCC1 in the Developing Hypothalamus
}

\author{
Bridget M. Nugent, ${ }^{1}$ Carla V. Valenzuela, ${ }^{4}$ Timothy J. Simons, ${ }^{3}$ and Margaret M. McCarthy ${ }^{1,2}$ \\ ${ }^{1}$ Program in Neuroscience, ${ }^{2}$ Departments of Physiology and Psychology, ${ }^{3}$ University of Maryland, School of Medicine, Baltimore, Maryland 21201, and \\ ${ }^{4}$ University of Maryland Baltimore County, Baltimore, Maryland 21250
}

In immature neurons the amino acid neurotransmitter, GABA provides the dominant mode for neuronal excitation by inducing membrane depolarization due to $\mathrm{Cl}^{-}$efflux through $\mathrm{GABA}_{\mathrm{A}}$ receptors $\left(\mathrm{GABA}_{\mathrm{A}} \mathrm{Rs}\right)$. The driving force for $\mathrm{Cl}^{-}$is outward because the $\mathrm{Na}^{+}$$\mathrm{K}^{+}-2 \mathrm{Cl}^{-}$cotransporter (NKCC1) elevates the $\mathrm{Cl}^{-}$concentration in these cells. GABA-induced membrane depolarization and the resulting activation of voltage-gated $\mathrm{Ca}^{2+}$ channels is fundamental to normal brain development, yet the mechanisms that regulate depolarizing GABA are not well understood. The neurosteroid estradiol potently augments depolarizing GABA action in the immature hypothalamus by enhancing the activity of the NKCC1 cotransporter. Understanding how estradiol controls NKCC1 activity will be essential for a complete understanding of brain development. We now report that estradiol treatment of newborn rat pups significantly increases protein levels of two kinases upstream of the NKCC1 cotransporter, SPAK (STE20/SPS1-related proline alanine rich kinase) and OSR1 (oxidative stress response kinase). The estradiol-induced increase is transcription dependent, and its time course parallels that of estradiol-enhanced phosphorylation of NKCC1. Antisense oligonucleotide-mediated knockdown of SPAK, and to a lesser degree of OSR1, precludes estradiol-mediated enhancement of NKCC1 phosphorylation. Functionally, knockdown of SPAK or OSR1 in embryonic hypothalamic cultures diminishes estradiol-enhanced $\mathrm{Ca}^{2+}$ influx induced by $\mathrm{GABA}_{\mathrm{A}} \mathrm{R}$ activation. Our data suggest that SPAK and OSR 1 may be critical factors in the regulation of depolarizing GABA-mediated processes in the developing brain. It will be important to examine these kinases with respect to sex differences and developmental brain anomalies in future studies.

\section{Introduction}

The principal inhibitory neurotransmitter in the adult brain, GABA, serves as the major source of neuronal excitation in the developing brain (Cherubini et al., 1991) and acts as a trophic factor during brain development by influencing cell survival (Obata, 1997; Luk and Sadikot, 2001), cell migration (Barker et al., 1998; Bolteus and Bordey, 2004), neurite outgrowth (Barbin et al., 1993; Maric et al., 2001), and synaptogenesis (Mitchell and Redburn, 1996; Belhage et al., 1998). GABA's excitatory effects on immature neurons are the result of a marked difference in cell membrane potential generated by the transmembrane chloride $\left(\mathrm{Cl}^{-}\right)$gradient. In a mature neuron, intracellular $\mathrm{Cl}^{-}$concentrations $\left(\left[\mathrm{Cl}^{-}\right]_{\mathrm{i}}\right)$ are low relative to $\left[\mathrm{Cl}^{-}\right]_{\mathrm{o}}$, creating a negative reversal potential for GABA in relation to the cell's resting

Received 0ct. 26, 2011; accepted Nov. 13, 2011.

Author contributions: B.M.N. and M.M.M. designed research; B.M.N., C.V.V., and T.J.S. performed research; B.M.N. analyzed data; B.M.N. and M.M.M. wrote the paper.

The authors declare no competing financial interests.

This work was supported by Grant R01 NS050525 to M.M.M. and Grant F31 NS073545-01 to B.M.N. We thank Dr. James Wade (University of Maryland, Baltimore, MD) for his helpful suggestions and generosity. We also thank Dr. Eric Delpire (Vanderbilt University, Nashville, TN) and Dr. Biff Forbush (Yale University, New Haven, CT) for generously providing us with the OSR1 and pNKCC1 antibodies used in this paper. In addition, we thank Dr. G. Michael Bowers, Dr. Jaylyn Waddell, and Dr. Bruce Krueger for assistance with Ca ${ }^{2+}$ imaging and Dr. Bradley Alger for his helpful suggestions for preparation of this manuscript.

Correspondence should be addressed to Bridget M. Nugent, Program in Neuroscience, University of Maryland, School of Medicine, 655 W. Baltimore Street, Room 5-014, Baltimore, MD 21201. E-mail: Bnuge001@ umaryland.edu.

DOI:10.1523/JNEUROSCI.5415-11.2012

Copyright $\odot 2012$ the authors $\quad 0270-6474 / 12 / 320593-06 \$ 15.00 / 0$ membrane potential, resulting in $\mathrm{Cl}^{-}$influx and membrane hyperpolarization. In immature cells, $\left[\mathrm{Cl}^{-}\right]_{\mathrm{i}}$ is high, which leads to $\mathrm{Cl}^{-}$efflux and membrane depolarization following $\mathrm{GABA}_{\mathrm{A}}$ receptor $\left(\mathrm{GABA}_{\mathrm{A}} \mathrm{R}\right)$ activation (LoTurco et al., 1995; Ben-Ari, 2002). The magnitude of membrane depolarization is frequently large enough to initiate opening of L-type voltage-dependent calcium channels (Obrietan and van den Pol, 1995), and the resulting $\mathrm{Ca}^{2+}$ influx mediates the trophic actions of GABA on the developing brain (Cruzalegui and Bading, 2000; Greer and Greenberg, 2008).

Despite its fundamental importance to neuronal maturation, relatively few variables have been indentified that modulate either the magnitude or frequency of depolarizing GABA effects. Depolarizing GABA activity peaks around the day of birth [postnatal day 0 ( $\mathrm{PN} 0)$ ] in the hypothalamus and gradually switches to inhibitory by PN6 (Obrietan and van den Pol, 1995). Estradiol is a gonadal steroid found at high levels in the developing brain of both sexes but significantly higher in select regions of the male rat brain, in particular the hypothalamus (McCarthy and Arnold, 2011). Most studies examining depolarizing GABA do not include physiological levels of estradiol yet this steroid significantly increases both the amplitude and likelihood of $\mathrm{Ca}^{2+}$ transients following $\mathrm{GABA}_{\mathrm{A}}$ activation and substantially delays the developmental switch from depolarizing to hyperpolarizing GABA (Perrot-Sinal et al., 2001). The absence of estradiol may cause an underestimate of depolarizing GABA effects in some experimental studies.

The activity of membrane-bound cation-chloride cotransporters establishes and maintains $\left[\mathrm{Cl}^{-}\right]_{\mathrm{i}}$. The activity of the $\mathrm{Na}^{+}{ }_{-}$ 
$\mathrm{K}^{+}-2 \mathrm{Cl}^{-}$cotransporter (NKCC1), which simultaneously moves $\mathrm{Na}^{+}, \mathrm{K}^{+}$and two $\mathrm{Cl}^{-}$ions across the membrane into the cell, is controlled by phosphorylation of a cluster of threonine residues located on its intracellular N-terminal tail (Vitari et al., 2005). Activity of the $\mathrm{K}^{+}-\mathrm{Cl}^{-}$cotransporter (KCC2) counterbalances NKCC1, by extruding ions from the cell. As expression levels of NKCC1 decrease during brain maturation, KCC2 expression correspondingly increases, gradually shifting GABA activity from depolarizing to hyperpolarizing (Plotkin et al., 1997; Lee et al., 2005). Estradiol does not affect the amount of these cotransporters in the hypothalamus, but instead markedly increases phosphorylation of NKCC1 at Thr212 and Thr217 (Perrot-Sinal et al., 2007), suggesting estradiol-mediated enhancement of depolarizing GABA involves controlling the activity of the NKCC1 cotransporter.

NKCC1 interacts with and is activated by the serine/threonine kinases STE20/ SPS1-related proline alanine rich kinase (SPAK; also known as PASK) and oxidative stress response kinase (OSR1) (Piechotta et al., 2002; Geng et al., 2009). SPAK and OSR1 have high sequence homology within their regulatory and catalytic domains and their expression often overlaps in the brain (Tamari et al., 1999). To investigate the molecular mechanisms of NKCC1 regulation by estradiol, we have quantified pNKCC1, SPAK, and OSR 1 in the developing hypothalamus. We found that estradiol increases SPAK and OSR1 in a transcription-dependent manner which subsequently leads to increased phosphorylation of NKCC1. To distinguish the relative contribution of SPAK versus OSR1, we knocked down expression of each using antisense oligonucleotides and quantified NKCC1 phosphorylation following estradiol administration. Finally, we confirmed that the level of these kinases is critical to estradiol-enhanced $\mathrm{Ca}^{2+}$ influx following $\mathrm{GABA}_{\mathrm{A}} \mathrm{R}$ activation in immature hypothalamic neurons. Congenital defects in steroidogenesis or environmental exposure to endocrine disrupting compounds can negatively impact brain development, yet the mechanisms by which these effects occur is largely unknown. Our results suggest a novel mode of estradiol action that may have important consequences for both normal and abnormal brain development.

\section{Materials and Methods}

Animals and injection procedures. Rat pups birthed by Sprague Dawley dams mated in the University of Maryland School of Medicine Animal Care Facility were housed under a $12 \mathrm{~h}$ reverse light/dark cycle and allowed ad libitum access to food and water. Drugs were administered as follows: estradiol benzoate (Sigma; $100 \mu \mathrm{g}$ in $0.1 \mathrm{ml}$, s.c., in sesame oil), actinomycin D (Sigma, $1 \mathrm{~mm}$ in $1 \mu$ l, bilateral, i.c.v.. in 5\% DMSO), cycloheximide (Sigma, $18 \mu \mathrm{M}$ in $0.1 \mathrm{cc}$, i.p., in 5\% DMSO), on the day of birth (PN0). Corresponding vehicle treatments were given in equivalent route and volume. Antisense oligonucleotides $(1 \mu \mathrm{g}$ of DNA in $1 \mu \mathrm{l}$ of $0.9 \%$ saline) were administered via bilateral i.c.v. injection to female rat pups on PN0 and PN1. Infusions were performed with a 23 gauge $1 \mu \mathrm{l}$ Hamilton syringe under cryoanesthesia, $1 \mathrm{~mm}$ caudal to bregma and 1 $\mathrm{mm}$ lateral to the midline to a depth of $2 \mathrm{~mm}$. All procedures were approved by the Institutional Animal Care and Use Committee of the University of Maryland, Baltimore.

Antisense oligonucleotides. Locked nucleic acid (LNA)-containing oligonucleotides were synthesized by Exiqon. Oligonucleotide sequences were generated from GenBank accession numbers for SPAK (NM_019362) and OSR1 (NM_001108194) mRNA. A random sequence [Scrambled (SCRAM)] oligonucleotide containing similar A-T and C-G content served as a control for oligonucleotide infusion. Sequences were as follows: OSR1, $\mathrm{CCT}^{\star} \mathrm{CGG}^{\star} \mathrm{ACAT}^{\star} \mathrm{GAC}^{\star} \mathrm{GGC}^{\star} \mathrm{TGC}$ (bp 286-304); SPAK, GG* ${ }^{\star}$ TCC $^{\star} \mathrm{GCC}^{\star} \mathrm{ATG}^{\star} \mathrm{ATGC}^{\star} \mathrm{TGC}$ (bp 409-427); SCRAM, $\mathrm{CGC}^{\star} \mathrm{TCG}^{\star} \mathrm{ATCC}^{\star} \mathrm{AGG}^{\star} \mathrm{TCA}^{\star}{ }^{\mathrm{GCG}}\left({ }^{*}\right.$ denotes position of locked nucleic acids). NCBI BLAST database query confirmed that each oligonucleotide had sequence homology only to its intended target sequence. Antisense oligonucleotide efficacy was assessed via Western immunoblot and resulted in a $22 \%$ decrease in SPAK protein, and a $24 \%$ decrease in OSR1 protein, which is in the typical range of antisense oligonucleotide knockdown (McCarthy et al., 2000). Antisense knockdown of SPAK had no effect on OSR1 protein levels and vice versa (data not shown).

Tissue collection. Brains were placed in a Zivic Miller brain block, with the ventral surface facing upward. To isolate the medial basal hypothalamus (MBH) a $2 \mathrm{~mm}$ coronal section was collected using the caudal boundary of the optic chiasm as a guide. The third ventricle was used to guide further microdissection of the MBH: $2 \mathrm{~mm}$ dorsal of the ventral surface of the brain, and $1 \mathrm{~mm}$ lateral in each direction from the third ventricle. Isolated tissue was frozen on dry ice and stored at $-80^{\circ} \mathrm{C}$ until use.

Western immunoblotting. Tissue was homogenized in RIPA buffer and Western immunoblotting was performed as previously described (Amateau and McCarthy, 2002) using anti-SPAK (Cell Signaling Technology, 1:1000), OSR1 (a generous gift from E. Delpire, Vanderbilt University, Nashville, TN, 1:5000), NKCC1 (Millipore, 1:1000), and pNKCC1 (a 

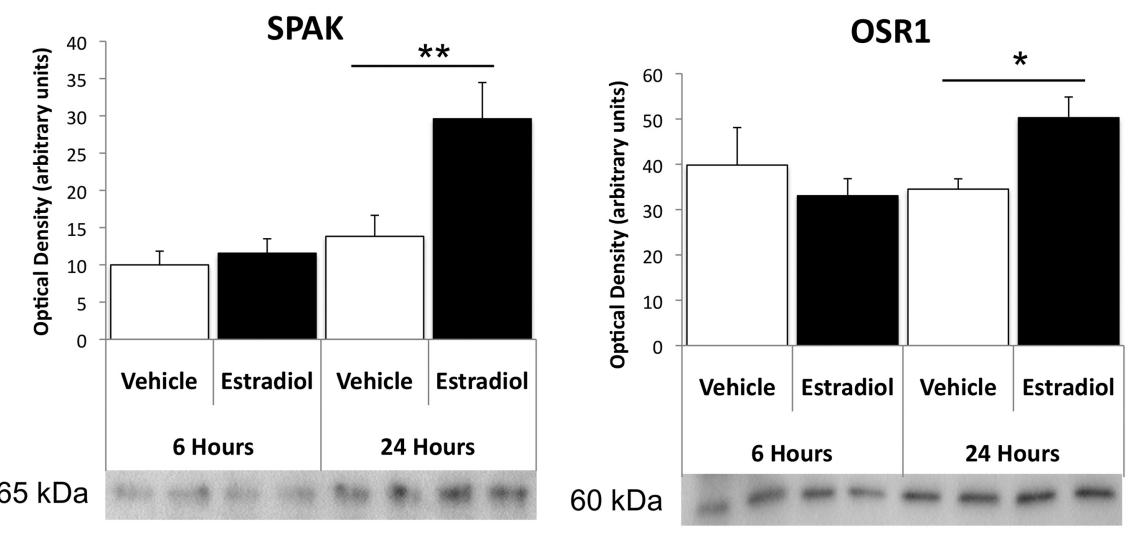

Figure 2. SPAK and OSR1 protein levels in the neonatal hypothalamus are increased $24 \mathrm{~h}$ after estradiol treatment. Levels of the serine/threonine kinases SPAK and OSR1 were quantified in the hypothalamus by Western immunoblot. In the same animals as used in Figure 1, SPAK or 0SR1 protein levels were not changed $6 \mathrm{~h}$ after estradiol treatment, but were significantly increased in the steroid-treated animals $24 \mathrm{~h}$ postinjection on PN1 (ANOVA, ${ }^{*} p<0.05,{ }^{* *} p<0.01$ ).
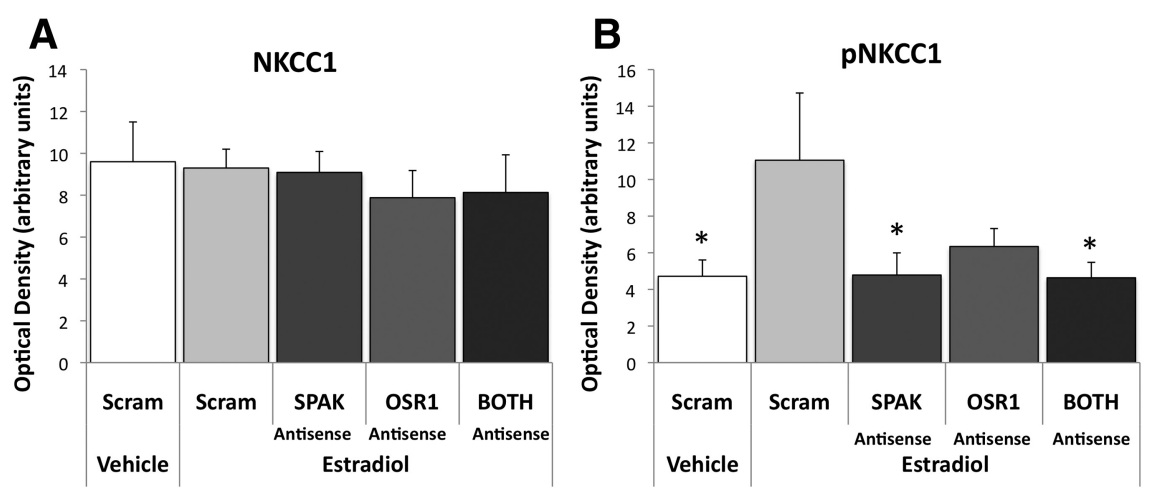

Figure 3. Antisense oligonucleotide knockdown of SPAK and ORS1 prevents estradiol-mediated enhancement of NKCC1 phosphorylation. NKCC1 and pNKCC1 in the hypothalamus were quantified by Western immunoblot following antisense oligo and estradiol treatment. $A$, Administration of antisense oligos against SPAK, OSR1, or BOTH had no effect on NKCC1 protein levels (ANOVA, $p=0.95$ ). $\boldsymbol{B}$, In animals treated with the control oligo (SCRAM), there was a significant increase in pNKCC1 levels in response to estradiol treatment compared with vehicle treatment (ANOVA, $p=0.026$ ). In animals treated with antisense oligos against SPAK ( $p=0.028$ ) or SPAK + OSR1 (BOTH; $p=0.025)$, estradiol-mediated enhancement of NKCC 1 phosphorylation was precluded. Administration of antisense oligos against OSR1 produced a trend in reduction of estradiol-mediated enhancement of NKCC1 phosphorylation compared with SCRAM + estradiol-treated control $(p=0.08) .{ }^{*} p<0.05$ compared with SCRAM + estradiol.

generous gift from B. Forbush, Yale University, New Haven, CT, 1:2500) antibodies followed by an anti-rabbit HRP-linked secondary (Cell Signaling Technology, 1:3000). Integrative grayscale pixel area densitometry was quantified using NIH Image software. Ponceau-S staining was used to standardize protein levels for all experiments. In experiments using actinomycin and cycloheximide, pNKCC1 levels were also normalized to total NKCC1 protein levels. Data were analyzed by ANOVA followed by Fisher's PLSD, where appropriate, with StatView (SAS Institute) software using $p<0.05$ as a criterion for statistical significance.

Cell culture and treatments. Hypothalamic cultures were generated from embryonic day 16 embryos as previously described (Perrot-Sinal et al., 2001). Cultures were maintained at $37^{\circ} \mathrm{C}$ in $5 \% \mathrm{CO}_{2}$ for $5 \mathrm{~d}$ until imaging was completed on day in vitro 4 (DIV 4). Cells were treated $24 \mathrm{~h}$ after plating on DIV 1 with the following: (1) vehicle (DMSO at final concentration of $0.01 \%$; Sigma $)+$ SCRAM oligo $(50 \mu \mathrm{M})$, (2) estradiol $\left(10^{-10} \mathrm{M}\right.$ final concentration) + SCRAM oligo, (3) estradiol + SPAK antisense oligo $(50 \mu \mathrm{M}),(4)$ estradiol + OSR1 antisense oligo $(50 \mu \mathrm{M})$, (5) estradiol + SPAK + OSR1 antisense oligos ( $25 \mu \mathrm{M}$ each). Cultures were treated with the same oligo again on DIV 3 during media change. Sterile culture medium was replaced by physiological saline solution (PSS; in mM: $134 \mathrm{NaCl}, 25$ glucose, $5 \mathrm{KCl}, 1 \mathrm{MgCl}, 3 \mathrm{CaCl}, 10$ HEPES, pH 7.35) before imaging.
$\mathrm{Ca}^{2+}$ imaging. Imaging with Fura-2AM (3 $\mu \mathrm{M}$; Invitrogen) in DMSO $(<0.5 \%)$ was performed as previously described (Nuñez and McCarthy, 2007). Cell bodies were selected based on identification of healthy-looking neuronal phenotype (triangular shape with rounded edges and at lease one process) and imaged individually at $60 \times$. Ratiometric fluorescent images were obtained every $10 \mathrm{~s}$. Emission at $510 \mathrm{~nm}$ following excitation at 340 and $380 \mathrm{~nm}$ wavelengths was used to calculate an $F_{340} / F_{380}$ fluorescence ratio. Following establishment of a stable baseline, $10 \mu \mathrm{M}$ muscimol (Sigma) was perfused for $50 \mathrm{~s}$, followed by PSS perfusion to ensure that signals returned to baseline (typically within $2-4 \mathrm{~min}$ ). As a positive control, cells were then perfused with 50 $\mathrm{mm} \mathrm{KCl}$ to ensure cell vitality. A total of 196 neurons from 15 cultures were imaged using a Zeiss Axiovert 100 inverted microscope. Fluorescent images were captured by a Hamamatsu CCD camera and MetaMorph/Metafluor Imaging System (Universal Imaging) as previously described (Waddell et al., 2011). When indicated, the NKCC1 blocker bumetanide (Sigma; $10 \mu \mathrm{M}$ in PSS) was perfused onto cultures as previously described (Choi et al., 2008).

During each imaging session the following parameters were quantified: (1) average baseline ratiometric signal from Fura-2AM excitation (to indicate basal intercellular $\mathrm{Ca}^{2+}$ levels), (2) Fura2AM excitation following muscimol application, and (3) number of cells responding with increased calcium transients (defined as a $10 \%$ or greater increase from baseline) following drug application.

\section{Results}

Estradiol-induced increases in NKCC1 phosphorylation are dependent upon transcription

Estradiol binds to a nuclear transcription factor receptor capable of diverse actions at the membrane and the genome. Estrogen receptors associated with the cell membrane rapidly activate kinase-mediated intracellular signaling pathways while the same receptors are capable of direct transcriptional regulation (McEwen, 1991; Beato et al., 1995). To distinguish between rapid versus genomic actions of estradiol on NKCC1 phosphorylation, we quantified pNKCC1 following relatively short-term $(6 \mathrm{~h})$ and long-term $(24 \mathrm{~h})$ estradiol exposure. Following $6 \mathrm{~h}$ of estradiol treatment there was no significant change in the amount of pNKCC1 measured by Western immunoblot, however $24 \mathrm{~h}$ later there was significantly increased pNKCC1 protein in the hypothalamus (ANOVA, $F_{(3,17)}=3.595, p=0.035$ main effect; Fisher's PLSD $p=0.02$ ) (Fig. $1 A$ ). We observed no change in total NKCC1 protein at either time point (Fig. $1 B$ ), confirming previous observations that estradiol enhances depolarizing GABA by controlling NKCC1 activity not the amount of the cotransporter (Perrot-Sinal et al., 2007). The requirement for long-term estradiol exposure suggests the activated estrogen receptor is not increasing cotransporter activity by rapidly or directly activating signaling pathways, but instead is probably influencing NKCC1 activity via genomic upregulation of specific kinases. To test this 

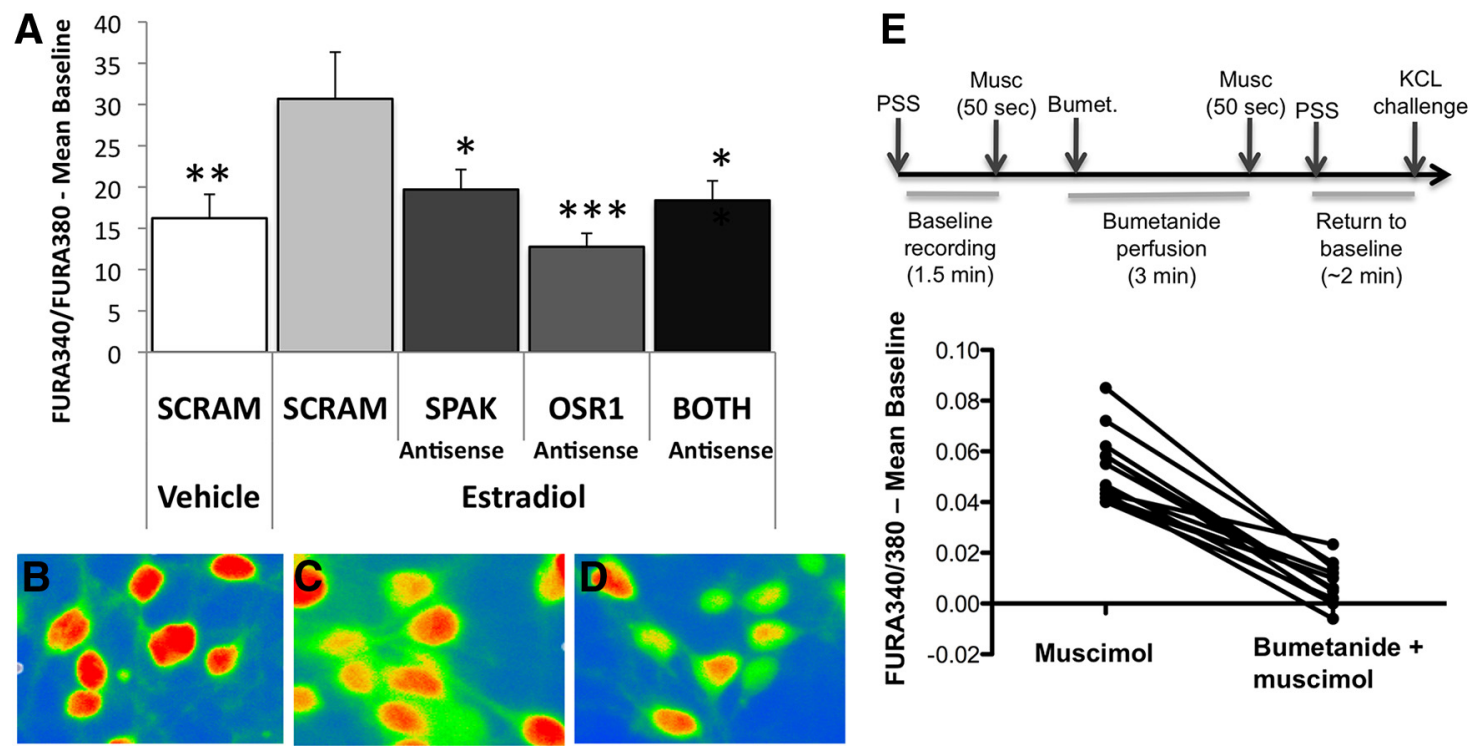

Figure 4. SPAK and ORS1 knockdown prevents estradiol-mediated enhancement of cellular responses to muscimol. Embryonic hypothalamic cultures were treated for $3 \mathrm{~d}$ with vehicle (DMSO) or $10^{-10} \mathrm{M}$ estradiol in addition to SCRAM, SPAK, OSR1, or BOTH antisense oligonucleotides and imaged on DIV 4 following incubation in the calcium-sensitive dye, Fura-AM. After establishment of baseline FURA340/FURA380 ratios, cultures were perfused with $10 \mu \mathrm{m}$ muscimol for $50 \mathrm{~s}$. A, Knockdown of SPAK, OSR1, or BOTH kinases prevented estradiol-mediated increases in [Ca $\left.{ }^{2+}\right]_{\mathrm{i}}($ ANOVA, $p=0.004) .{ }^{*} p<0.05,{ }^{* *} p<0.01,{ }^{* * *} p<0.001 . \boldsymbol{B}-\boldsymbol{D}$, Representative images of cells treated with Estradiol+SCRAM-oligo (B), Estradiol+SPAK antisense oligo (C), and Estradiol+0SR1 antisense oligo (D) captured 30 s after initiation of muscimol perfusion. $\boldsymbol{E}$, Blockade of the NKCC1 cotransporter with bumetanide eliminated estradiol-mediated enhancement of $\left[\mathrm{Ca}^{2+}\right]_{\mathrm{i}}$ in response to muscimol (paired $t$ test, $p<0.001$ ). Experimental design outlined above.

hypothesis we treated rat pups with either estradiol or vehicle in conjunction with the transcriptional and translational blockers actinomycin D and cycloheximide, respectively, before quantification of NKCC1 phosphorylation via Western blot. Blockade of transcription (ANOVA, $F_{(3,22)}=6.595, p=0.0024$; Fig. $\left.1 C\right)$ or translation (ANOVA, $F_{(3,23)}=5.754, p=0.0043$; Fig. $1 D$ ) prevented estradiol-induced increases in pNKCC1, supporting the hypothesis that estradiol enhances NKCC1 activity via upstream signaling proteins.

\section{Estradiol upregulates SPAK and OSR1}

The serine/threonine kinases SPAK and OSR1 are well known to phosphorylate and activate NKCC1 (Piechotta et al., 2002; Geng et al., 2009). We therefore hypothesized that estradiol enhances depolarizing GABA action by augmenting protein levels of SPAK and ORS1 upstream of NKCC1. Following $6 \mathrm{~h}$ of estradiol treatment, there were no significant changes in protein levels of SPAK or OSR1 in the developing hypothalamus. However, $24 \mathrm{~h}$ of estradiol treatment significantly increased SPAK (ANOVA, $F_{(3,16)}=8.817, p=0.001$ main effect; Fisher's PLSD, $p=0.003$ ) and OSR1 protein levels (ANOVA, $F_{(3,18)}=2.536, p=0.0892$ main effect; Fisher's PLSD, $p=$ 0.04; Fig. 2), precisely correlating with the estradiol-mediated time course for NKCC1 phosphorylation.

Both SPAK and OSR1 are activated by the WNK family of kinases (Vitari et al., 2005). To assess estradiol's effect on these family members, we performed Western immunoblots for WNK1, WNK3, and WNK4 as well as phosphorylated (activated) SPAK in the hypothalamus. We found no effect of estradiol on protein levels of WNK kinases or PSPAK following $24 \mathrm{~h}$ of estradiol treatment, indicating that estradiol does not affect NKCC1 signaling by acting upstream of SPAK and OSR1 (data not shown).
Knockdown of SPAK and OSR1 precludes estradiol-induced NKCC1 activation and enhanced calcium influx in response to $\mathrm{GABA}_{\mathrm{A}}$ activation

To determine the importance of SPAK and OSR1 in estradiolmediated enhancement of NKCC1 activation we knocked down SPAK and OSR1 expression using antisense oligonucleotides (oligos) directed against their respective mRNAs. We predicted that administration of antisense oligos against SPAK, OSR1, or both would preclude estradiol-induced phosphorylation of NKCC1 and prevent estradiol-induced enhancement of neuronal $\mathrm{Ca}^{2+}$ influx in response to muscimol, a selective $\mathrm{GABA}_{\mathrm{A}}$ agonist. In animals treated with estradiol, SPAK antisense oligos significantly reduced pNKCC1 compared with SCRAM oligo treatment (Fisher's PLSD, $p=0.028$ ), resulting in levels similar to those seen in control animals treated with vehicle and SCRAM-oligos, indicating that SPAK is necessary for estradiol-mediated enhancement of NKCC1 phosphorylation (ANOVA, $F_{(4,20)}=$ $2.170, p<0.05$ main effect; Fig. $3 B$ ). Knockdown of OSR1 caused a moderate trend toward decreasing pNKCC1 in the presence of estradiol ( $p=0.08$ ). Finally, the combination of both SPAK and OSR1 antisense oligos (BOTH) significantly reduced pNKCC1 levels compared with SCRAM-oligo control with estradiol on board ( $p=0.025)$. As expected, we saw no effect of antisense oligonucleotide-mediated knockdown of either kinase on total NKCC1 levels (ANOVA, $F_{(4,19)}=0.252, p=0.948$; Fig. $3 A$ ) and treatment with the control (SCRAM) oligo had no effect on phosphorylation of NKCC1, since PNKCC1 in SCRAM oligo-treated animals was higher in animals simultaneously treated with estradiol compared with vehicle ( $p=0.026$; Fig. $3 B$ ).

Consistent with previous reports (Perrot-Sinal et al., 2001), estradiol enhanced $\mathrm{Ca}^{2+}$ transient amplitude compared with vehicle treatment in response to the $\mathrm{GABA}_{\mathrm{A}}$ agonist muscimol in cultured embryonic hypothalamic neurons in the presence of SCRAM oligo (ANOVA, $F_{(4,57)}=3.915, p<0.0071$; Fisher's 
PLSD, $p=0.0039)$. Cultures treated with SPAK $(p=0.0129)$, OSR1 $(p=0.0004)$, or BOTH ( $p=0.0083)$ antisense oligos in conjunction with estradiol displayed significantly reduced $\mathrm{Ca}^{2+}$ transient amplitudes in response to muscimol as indicated by ratiometric Fura-2AM signal enhancement (Fig. 4A). Thus phosphorylation of the NKCC1 cotransporter by SPAK or OSR1 is necessary for estradiol-mediated enhancement of developmental excitatory GABA in hypothalamic neurons. Estradiol also increases the percentage of cells that respond to muscimol with detectable calcium influx (Perrot-Sinal et al., 2001). In the current experiment, $45 \%$ of vehicle/SCRAM oligo-treated cells responded to muscimol, whereas $72 \%$ of cells responded when treated with estradiol/SCRAM-oligo. Interestingly, knockdown of SPAK $(91 \%)$, OSR1 (85\%), or BOTH (60\%) did not decrease the percentage of cells responding in the presence of estradiol. Thus reduction of these kinases does not incapacitate GABAmediated depolarization, but significantly attenuates the magnitude of responsiveness to muscimol.

To determine whether NKCC1 activation is necessary for estradiol's enhancement of Fura-2AM ratiometric signals and rule out the possibility that estradiol treatment interferes with an unknown aspect of $\mathrm{Ca}^{2+}$ signaling, Fura ratios were recorded in response to muscimol in estradiol-treated neurons, before and after application of the NKCC1 blocker bumetanide. Elimination of NKCC1 activity with bumetanide completely blocked the ability of $\mathrm{GABA}_{\mathrm{A}}$ activation to alter $\mathrm{Ca}^{2+}$ transients in estradioltreated cultures (paired $t$ test, $t_{(12)}=11.56, p<0.001$ ).

\section{Discussion}

The discovery that the dominant inhibitory neurotransmitter, GABA, is also the major source of excitation in the developing brain was so surprising and unorthodox it required years of converging evidence from multiple laboratories to gain general acceptance (Ben-Ari, 2002) and continues to draw challenges some 20 years after the initial reports (Rheims et al., 2009; Waddell et al., 2011). Fundamental developmental endpoints regulated by depolarizing GABA action include giant depolarizing potentials (Ben-Ari et al., 1989), leading to spontaneous activity patterns (Blankenship and Feller, 2010), activity-dependent survival (Sauer and Bartos, 2010), neurite outgrowth (Sernagor et al., 2010), progenitor proliferation (Liu et al., 2005), and Hebbianbased synaptic patterning (Wang and Kriegstein, 2008). We previously identified an endogenous regulator of depolarizing GABA action, the gonadal and neurosteroid estradiol, which both amplifies the magnitude and extends the developmental duration of excitatory GABA (Perrot-Sinal et al., 2001). Estradiol is a pervasive signaling molecule that varies in concentration between brain regions, across development and in males versus females, thereby contributing to variability in neuronal maturation. The present studies reveal that this steroid enhances depolarizing GABA effects by increasing levels of the signaling kinases SPAK and OSR1, which are upstream of the NKCC1 cotransporter. Estradiol-mediated increases in NKCC1 phosphorylation are precluded by antisense oligonucleotide-mediated knockdown of SPAK, and to a lesser extent OSR1, exhibiting the necessity of these kinases for mediating estradiol's effects. Furthermore, knockdown of either or both of these kinases significantly attenuated estradiol's enhancement of intracellular $\mathrm{Ca}^{2+}$ influx in response to $\mathrm{GABA}_{\mathrm{A}}$ activation.

Estradiol has widespread effects on cellular processes through both rapid, nongenomic actions on cell signaling, and slower more enduring effects by modulating transcriptional activity (McEwen, 1991). The combination of a long time course and a complete ablation of the effectiveness of estradiol by simultaneous administration of blockers of transcription or translation confirm that the cascade of events leading to estradiol enhancement of depolarizing GABA begins with increased gene expression. The ability of the brain to synthesize estradiol in discrete loci raises the specter of estrogens as widespread endogenous regulators of depolarizing GABA actions that broadly impact on brain development.

Disregulation in developmental excitatory GABAergic signaling has been shown to impair the development of neuronal circuits and may be a contributing factor in neurodevelopmental disorders such as epilepsy, autism spectrum disorders, and schizophrenia (Briggs and Galanopoulou, 2011; Hyde et al., 2011; Pizzarelli and Cherubini, 2011). Sex differences have been widely reported in all of these disorders, implicating a role for estradiol in their etiology. Targeting SPAK or OSR1 may allow for novel therapeutic options for these neural disorders.

\section{References}

Amateau SK, McCarthy MM (2002) A novel mechanism of dendritic spine plasticity involving estradiol induction of prostaglandin-E2. J Neurosci 22:8586-8596.

Barbin G, Pollard H, Gaïarsa J, Ben-Ari Y (1993) Involvement of GABAA receptors in the outgrowth of cultured hippocampal neurons. Neurosci Lett 152:150-154.

Barker JL, Behar T, Li YX, Liu QY, Ma W, Maric D, Maric I, Schaffner AE, Serafini R, Smith SV, Somogyi R, Vautrin JY, Wen XL, Xian H (1998) GABAergic cells and signals in CNS development. Perspect Dev Neurobiol 5:305-322.

Beato M, Herrlich P, Schütz G (1995) Steroid hormone receptors: many actors in search of a plot. Cell 83:851-857.

Belhage B, Hansen GH, Elster L, Schousboe A (1998) Effects of gammaaminobutyric acid (GABA) on synaptogenesis and synaptic function. Perspect Dev Neurobiol 5:235-246.

Ben-Ari Y (2002) Excitatory actions of GABA during development: The nature of the nurture. Nat Rev Neurosci 3:728-739.

Ben-Ari Y, Cherubini E, Corradetti R, Gaiarsa JL (1989) Giant synaptic potentials in immature rat CA3 hippocampal neurones. J Physiol 416:303-325.

Blankenship AG, Feller MB (2010) Mechanisms underlying spontaneous patterned activity in developing neural circuits. Nat Rev Neurosci 11:18-29.

Bolteus AJ, Bordey A (2004) GABA release and uptake regulate neuronal precursor migration in the postnatal subventricular zone. J Neurosci 24:7623-7631.

Briggs SW, Galanopoulou AS (2011) Altered GABA signaling in early life epilepsies. Neural Plast. Advance online publication. Retrieved July 25, 2011. doi:10.1155/2011/527605.

Cherubini E, Gaiarsa JL, Ben-Ari Y (1991) GABA: An excitatory transmitter in early postnatal life. Trends Neurosci 14:515-519.

Choi HJ, Lee CJ, Schroeder A, Kim YS, Jung SH, Kim JS, Kim do Y, Son EJ, Han HC, Hong SK, Colwell CS, Kim YI (2008) Excitatory actions of GABA in the suprachiasmatic nucleus. J Neurosci 28:5450-5459.

Cruzalegui FH, Bading H (2000) Calcium-regulated protein kinase cascades and their transcription factor targets. Cell Mol Life Sci 57:402-410.

Geng Y, Hoke A, Delpire E (2009) The ste20 kinases SPAK and OSR1 regulate NKCC1 function in sensory neurons. J Biol Chem 284:14020-14028.

Greer PL, Greenberg ME (2008) From synapse to nucleus: calciumdependent gene transcription in control of synapse development and function. Neuron 59:846-860.

Hyde TM, Lipska BK, Ali T, Mathew SV, Law AJ, Metitiri OE, Straub RE, Ye T, Colantuoni C, Herman MM, Bigelow LB, Weinberger DR, Kleinman JE (2011) Expression of GABA signaling molecules KCC2, NKCC1, and GAD1 in cortical development and schizophrenia. J Neurosci 31:11088-11095.

Lee H, Chen CX, Liu YJ, Aizenman E, Kandler K (2005) KCC2 expression in immature rat cortical neurons is sufficient to switch the polarity of GABA responses. Eur J Neurosci 21:2593-2599.

Liu X, Wang Q, Haydar TF, Bordey A (2005) Nonsynaptic GABA signaling in postnatal subventricular zone controls proliferation of GFAPexpressing progenitors. Nat Neurosci 8:1179-1187. 
LoTurco JJ, Owens DF, Heath MJ, Davis MB, Kriegstein AR (1995) GABA and glutamate depolarize cortical progenitor cells and inhibit DNA synthesis. Neuron 15:1287-1298.

Luk K, Sadikot A (2001) GABA promotes survival but not proliferation of parvalbumin-immunoreactive interneurons in rodent neostriatum: an in vivo study with stereology. Neuroscience 104:93-103.

Maric D, Liu QY, Maric I, Chaudry S, Chang YH, Smith SV, Sieghart W, Fritschy JM, Barker JL (2001) GABA expression dominates neuronal lineage progression in the embryonic rat neocortex and facilitates neurite outgrowth via GABAA Autoreceptor/Cl $\{-\}$ channels. J Neurosci 21:2343-2360.

McCarthy MM, Arnold AP (2011) Reframing sexual differentiation of the brain. Nat Neurosci 14:677-683.

McCarthy MM, Auger AP, Mong JA, Sickel MJ, Davis AM (2000) Antisense oligonucleotides as a tool in developmental neuroendocrinology. Methods 22:239-248.

McEwen BS (1991) Non-genomic and genomic effects of steroids on neural activity. Trends Pharmacol Sci 12:141-147.

Mitchell CK, Redburn DA (1996) GABA and GABA-A receptors are maximally expressed in association with cone synaptogenesis in neonatal rabbit retina. Brain Res Dev Brain Res 95:63-71.

Nuñez JL, McCarthy MM (2007) Evidence for an extended duration of GABA-mediated excitation in the developing male versus female hippocampus. Dev Neurobiol 67:1879-1890.

Obata K (1997) Excitatory and trophic action of GABA and related substances in newborn mice and organotypic cerebellar culture. Dev Neurosci 19:117-119.

Obrietan K, van den Pol AN (1995) GABA neurotransmission in the hypothalamus: developmental reversal from $\mathrm{Ca}^{2+}$ elevating to depressing. J Neurosci 15:5065-5077.

Perrot-Sinal TS, Davis AM, Gregerson KA, Kao JP, McCarthy MM (2001) Estradiol enhances excitatory gammabutyric acid-mediated calcium signaling in neonatal hypothalamic neurons. Endocrinology 142:22382243.

Perrot-Sinal TS, Sinal CJ, Reader JC, Speert DB, McCarthy MM (2007) Sex differences in the chloride cotransporters, $\mathrm{NKCC} 1$ and $\mathrm{KCC} 2$, in the developing hypothalamus. J Neuroendocrinol 19:302-308.

Piechotta K, Lu J, Delpire E (2002) Cation chloride cotransporters interact with the stress-related kinases Ste20-related proline-alanine-rich kinase (SPAK) and oxidative stress response 1 (OSR1). J Biol Chem 277:50812-50819.

Pizzarelli R, Cherubini E (2011) Alterations of GABAergic signaling in autism spectrum disorders. Neural Plast. Advance online publication. Retrieved July 25, 2011. doi:10.1155/2011/527605.

Plotkin MD, Snyder EY, Hebert SC, Delpire E (1997) Expression of the $\mathrm{Na}-\mathrm{K}-2 \mathrm{Cl}$ cotransporter is developmentally regulated in postnatal rat brains: A possible mechanism underlying GABA's excitatory role in immature brain. J Neurobiol 33:781-795.

Rheims S, Holmgren CD, Chazal G, Mulder J, Harkany T, Zilberter T, Zilberter Y (2009) GABA action in immature neocortical neurons directly depends on the availability of ketone bodies. J Neurochem 110:1330-1338.

Sauer JF, Bartos M (2010) Recruitment of early postnatal parvalbuminpositive hippocampal interneurons by GABAergic excitation. J Neurosci 30:110-115.

Sernagor E, Chabrol F, Bony G, Cancedda L (2010) GABAergic control of neurite outgrowth and remodeling during development and adult neurogenesis: general rules and differences in diverse systems. Front Cell Neurosci 4:11.

Tamari M, Daigo Y, Nakamura Y (1999) Isolation and characterization of a novel serine threonine kinase gene on chromosome 3p22-21.3. J Hum Genet 44:116-120.

Vitari AC, Deak M, Morrice NA, Alessi DR (2005) The WNK1 and WNK4 protein kinases that are mutated in Gordon's hypertension syndrome phosphorylate and activate SPAK and OSR1 protein kinases. Biochem J 391:17-24.

Waddell J, Kim J, Alger BE, McCarthy MM (2011) The depolarizing action of GABA in cultured hippocampal neurons is not due to the absence of ketone bodies. PloS One 6:e23020.

Wang DD, Kriegstein AR (2008) GABA regulates excitatory synapse formation in the neocortex via NMDA receptor activation. J Neurosci 28:55475558 . 